\section{Psicologia Escolar \\ e Educacional}

ARTIGO

DOI: http://dx.doi.org/10.1590/2175-35392021224238

Localizador - e224238

\title{
ADAPTAÇÃO E SAÚDE MENTAL DO ESTUDANTE UNIVERSITÁRIO: REVISÃO SISTEMÁTICA DA LITERATURA
}

\author{
Fernanda Torres Sahão ${ }^{1} \mathbb{D}$; Nádia Kienen ${ }^{1} \mathbb{D}$
}

RESUMO

A saúde mental do estudante e sua adaptação à universidade têm sido investigados em diversas pesquisas. O presente estudo teve como objetivo realizar revisão sistemática da literatura acerca das variáveis relativas à adaptação do estudante ao ensino superior que influenciam a sua saúde mental. Foi realizada busca bibliográfica em seis bases de dados nacionais e internacionais, sendo selecionados 23 artigos para análise a partir de seis categorias: dificultadores e facilitadores da adaptação, consequências da não adaptação, sintomas, repertório requerido e estratégias de ensino. Os resultados indicam que os principais dificultadores são nível de exigência e relacionamentos interpessoais; como facilitadores, rede de apoio, fornecimento de informação e integração acadêmica. Ansiedade, estresse e depressão são os principais sintomas; repertórios de autonomia e comprometimento os mais requeridos para uma adaptação saudável à universidade. Há carência de pesquisas que caracterizem os comportamentos constituintes desses repertórios, o que possibilitaria intervenções com essa população.

Palavras-chave: ensino superior; ajustamento; revisão de literatura.

\section{University student adaptation and mental health: a systematic review of literature}

\begin{abstract}
The student's mental health and his/her adaptation to the university have been investigated in several studies. The present study aimed to carry out a systematic review of the literature about the variables related to the student's adaptation to higher education that influence his/her mental health. A bibliographic search was carried out in six national and international databases, with 23 articles selected for analysis from six categories: difficulties and facilitators of adaptation, consequences of non-adaptation, symptoms, required repertoire and teaching strategies. The results indicate that the main obstacles are the level of demand and interpersonal relationships; as facilitators, support network, information provision and academic integration. Anxiety, stress and depression are the main symptoms; repertoires of autonomy and commitment most required for a healthy adaptation to the university. There is a lack of research that characterizes the behaviors that make up these repertoires, which would allow interventions with this population.
\end{abstract}

Keywords: higher education; adjustment; literature review.

\section{Adaptación y salud mental del estudiante universitario: revisión sistemática de la literatura}

\section{RESUMEN}

La salud mental del estudiante y su adaptación a la universidad han sido investigados en diversas investigaciones. En el presente estudio se tuvo como objetivo realizar revisión sistemática de la literatura acerca de las variables relativas a la adaptación del estudiante a la enseñanza universitaria que influencian su salud mental. Se realizó búsqueda bibliográfica en seis bases de datos nacionales e internacionales, siendo seleccionados 23 artículos para análisis a partir de seis categorías: dificultadores y facilitadores de la adaptación, consecuencias de la no adaptación, síntomas, repertorio requerido y estrategias de enseñanza. Los resultados indican que los principales dificultadores son nivel de exigencia y relacionamientos interpersonales; como facilitadores, red de apoyo, suministro de información e integración académica. Ansiedad, estrés y depresión son los principales síntomas; repertorios de autonomía y comprometimiento los más requeridos para una adaptación saludable a la universidad. Hay escasez de investigaciones que caractericen los comportamientos constituyentes de esos repertorios, lo que posibilita intervenciones con esa populación.

Palabras clave: enseñanza universitaria; adaptación; revisión de literatura.

$\overline{1}$ Universidade Estadual de Londrina - Londrina - PR - Brasil; ftsahao@gmail.com; nadiakienen@gmail.com 


\section{INTRODUÇÃO}

A saúde mental do estudante e sua adaptação à universidade têm sido objeto de estudo de diversas pesquisas, porém, essa literatura ainda apresenta lacunas importantes. Estudos apontam uma taxa mais alta de prevalência de sintomas de ansiedade e depressão na população universitária do que na população geral, acusando, inclusive, aumento dessa taxa nos últimos anos (Padovani et al., 2014). Conforme pesquisa realizada sobre perfil socioeconômico dos estudantes de universidades federais (FONAPRACE, 2014), 58,36\% do total de estudantes pesquisados indicaram sofrer de ansiedade; $44,72 \%$ relataram desânimo/falta de vontade de fazer as coisas; 32,57\% têm insônia ou apresentam alterações significativas de sono; $22,55 \%$ relataram sentir desamparo/desespero/ desesperança e 21,29\% sentimentos de solidão. Além dessas dificuldades, também são descritos problemas alimentares (13\%), medo/pânico $(10,56 \%)$, ideia de morte $(6,38 \%)$ e pensamento suicida $(4,13 \%)$.

O sofrimento mental dos estudantes universitários parece estar relacionado a uma série de demandas características do contexto de ingresso na universidade. Tais demandas envolvem a necessidade de adaptação relacionada à moradia, ao suporte social, à autonomia, às exigências relacionadas ao conteúdo abordado nas disciplinas, entre outras (Padovani et al., 2014). Estudos internacionais e nacionais também indicam uma preocupação de gestores e professores das universidades com relação à grande incidência de insucesso acadêmico dos alunos, manifestada de diversos modos, como baixas classificações, absenteísmo, mudanças de curso e abandonos (Oliveira \& Dias, 2014; Withey, Fox, \& Hartley, 2014).

Diversos estudos em que são investigados fenômenos relacionados à adaptação do estudante e sua saúde mental (e.g., Bore, Pittolo, Kirby, Dluzewska, \& Marlin, 2016; Oliveira \& Dias, 2014) destacam a necessidade de realização de pesquisas e intervenções com a população universitária. Isso porque a transição para a vida acadêmica parece ser uma experiência potencialmente estressora para jovens estudantes, o que pode influenciar no modo como eles aproveitarão as oportunidades oferecidas pela universidade, tanto com relação à formação profissional quanto ao desenvolvimento psicossocial. A maior parte dos estudos na área trata da identificação das dificuldades encontradas pelos estudantes (Moreno \& Soares, 2014; Padovani et al., 2014) ou de sintomas por eles apresentados, destacando diagnósticos como "ansiedade", "estresse" e "depressão" (Mahmoud, Staten, Hall, \& Lennie, 2012; Persaud \& Persaud, 2016). Essas informações, apesar de serem importantes para o conhecimento da realidade dessa população e para problematizar o fenômeno em questão, não descrevem ou caracterizam quais os repertórios específicos que os estudantes necessitam desenvolver para manejarem adequadamente tais situações, nem descrevem como são feitas as intervenções nesse contexto, como formas de amenizar ou solucionar tal situação.

Apesar de alguns estudos sinalizarem variáveis relativas aos comportamentos que os estudantes devem apresentar para se adaptarem ao contexto acadêmico (e.g., Bore et al., 2016; Stoliker \& Lafreniere, 2015), esses ainda as explicitam de forma pouco sistematizada e, muitas vezes, fazendo uso de nomenclaturas distintas para referirem-se aos mesmos fenômenos. Um exemplo é a variável relacionada ao contato social entre os estudantes nas primeiras semanas, que é apresentada como "integração com os estudantes", "inserção social" (Teixeira, Dias, Wottrich, \& Oliveira, 2008), "acolhimento dos estudantes" (Oliveira, Santos, \& Dias, 2016) e "amizades estabelecidas" (Oliveira \& Dias, 2014).

Sistematizar as variáveis descritas na literatura, buscando identificar e descrever aspectos relativos aos dificultadores e facilitadores para a adaptação, às consequências desses para a saúde mental dos estudantes, ao repertório requerido deles para que se adaptem de forma saudável ao contexto universitário, assim como as estratégias de ensino desse repertório pode contribuir para (1) dar consistência ao que já se sabe sobre a adaptação do estudante ao ensino superior, (2) evidenciar lacunas que ainda precisam ser investigadas, assim como (3) fundamentar o desenvolvimento de futuras intervenções. Ao revisar a literatura sobre tal fenômeno, foi encontrada apenas uma revisão sistemática que visou investigar a relação entre o uso de estratégias de coping e adaptação de estudantes universitários (Oliveira, Carlotto, Vasconcelos, \& Dias, 2014). Isso demonstra a necessidade de uma revisão sistemática mais ampla, que explicite outros aspectos envolvidos na adaptação ao contexto universitário, tais como os anteriormente citados. Dessa forma, este estudo teve como objetivo realizar uma revisão sistemática da literatura acerca das variáveis relativas à adaptação do estudante ao ensino superior que influenciam a sua saúde mental.

\section{MÉTODO}

Para responder ao objetivo proposto, foi realizada uma revisão sistemática da literatura, um método que objetiva identificar o maior número possível de estudos sobre um determinado fenômeno de forma organizada, resultando em uma análise reflexiva, crítica e compreensiva a respeito do material selecionado (Costa \& Zoltowski, 2014).

A busca bibliográfica foi realizada em seis bases de dados nacionais e internacionais: INDEXPSI, SCIELO, LILACS, PSYCNET, PUBMED e ERIC. Nas bases nacionais foram utilizados os descritores: "saúde mental" E estudante, universitário; ajustamento, acadêmico $E$ 
estudante; ensino superior $\mathrm{E}$ adaptação. Nas bases internacionais os descritores utilizados foram: mental health, undergraduate students AND adaptation; mental health, undergraduate students AND adjustment.

Foram incluídos artigos empíricos que apresentavam informações sobre (a) principais dificuldades encontradas pelos estudantes na transição para o ensino superior; (b) como são realizadas as intervenções nesse contexto; (c) como os estudantes se adaptam às mudanças necessárias; (d) queixas apresentadas por estudantes do ensino superior com relação a esse contexto; (e) fatores que favorecem ou dificultam a adaptação ao ensino superior. Foram selecionados artigos em português, inglês e espanhol. Os critérios de exclusão utilizados foram: (a) estudos sobre saúde mental com outras populações; (b) estudos sobre transtornos específicos não relacionados ao processo de adaptação (e.g., TDAH, transtornos de aprendizagem); (c) dificuldades encontradas em cursos específicos (e.g., área da saúde, esportistas); (d) estudos que só apresentavam dados de prevalência, sem descrição das variáveis relativas à adaptação; (e) aspectos relacionados a diferenças de gênero, raça, preconceito; (f) estudos de evidência de validade de instrumentos psicométricos; (g) estudos teóricos; ( $h$ ) idiomas diferentes de português, inglês e espanhol. A definição das palavras-chave, critérios de inclusão e exclusão e seleção dos artigos para revisão foi feita por duas pesquisadoras.

O levantamento bibliográfico foi realizado de setembro a novembro de 2017, e uma nova busca para atualização da revisão foi realizada em outubro de 2020, na qual foram selecionados quatro novos artigos para incluir na revisão. No total, foram encontradas 925 publicações, das quais 139 foram selecionadas e 42 recuperadas. Os trabalhos encontrados foram selecionados a partir do exame dos títulos e palavraschave e, posteriormente, da leitura dos resumos. Após a leitura completa dos artigos, considerando os critérios de inclusão e exclusão, 23 referências foram incluídas na revisão (Tabela 1).

\section{INSTRUMENTOS E MATERIAIS}

Para registro dos dados, foi criada uma tabela

Tabela 1 - Estudos Selecionados Para a Revisão.

\begin{tabular}{|c|c|}
\hline Código & Referência completa \\
\hline Art. 1 & $\begin{array}{l}\text { Ababu, G. B.; Yigzaw, A. B.; Besene, Y. D.; Alemu, W. G. (2018). Prevalence of adjustment problem and its predictors } \\
\text { among first-year undergraduate students in Ethiopian university: A cross-sectional institution based study. } \\
\text { Psychiatry Journal, 2018, 1-7. doi: 10.1155/2018/5919743 }\end{array}$ \\
\hline Art. 2 & $\begin{array}{l}\text { Barbosa, M. M. F.; Oliveira, M.C.; Melo-Silva, L. L.; Taveira, M. C. (2018). Delineamento e avaliação de um programa } \\
\text { de adaptação acadêmica no ensino superior. Revista Brasileira de Orientação Profissional, 19(1), 61-74. doi: } \\
\text { 1026707/1984-7270/2019v19n1p61 }\end{array}$ \\
\hline Art. 3 & $\begin{array}{l}\text { Bore, M., Pittolo; C., Kirby, D.; Dluzewska, T.; Marlin, S. (2016). Predictors of psychological distress and well-being in } \\
\text { a sample of Australian undergraduate students. Higher Education Research \& Development, 35(5), 869-880. doi: } \\
10.1080 / 07294360.2016 .1138452\end{array}$ \\
\hline Art. 4 & $\begin{array}{l}\text { Fernández-Rodríguez, C.; Soto-López, T.; Cuesta, M. (2019). Needs and demands for psychological care in university } \\
\text { students. Psicothema, 31(4), 414-421. doi:10.7334/psicothema2019.78 }\end{array}$ \\
\hline Art. 5 & $\begin{array}{l}\text { Hartley, M. T. (2011). Examining the relationships between resilience, mental health, and academic persistence in } \\
\text { undergraduate college students. Journal of American College Health, 59(7), 596-604. doi:10.1080/07448481.201 } \\
0.515632\end{array}$ \\
\hline Art. 6 & $\begin{array}{l}\text { Igue, E. A.; Bariani, I. C. B.; Milanesi, P. V. B. (2008). Vivência acadêmica e expectativas de universitários ingressantes } \\
\text { e concluintes. Psico-USF, 13(2), 155-164. doi: } 10.1590 / \text { S1413-82712008000200003 }\end{array}$ \\
\hline Art. 7 & $\begin{array}{l}\text { Klainin-Yobas, P.; Ramirez, D.; Fernandez, Z.; Sarmiento, J.; Thanoi, W.; Ignacio, J.; Lau, Y. (2016). Examining the } \\
\text { predicting effect of mindfulness on psychological well-being among undergraduate students: A structural } \\
\text { equation modelling approach. Personality and Individual Differences, 91,63-68. doi:10.1016/j.paid.2015.11.034 }\end{array}$ \\
\hline Art. 8 & $\begin{array}{l}\text { Levin, M. E.; Pistorello, J.; Seeley, J. R.; Hayes, S. C. (2014). Feasibility of a prototype web-based acceptance and } \\
\text { commitment therapy prevention program for college students. Journal of American College Health, 62(1), 20-30. } \\
\text { doi:10.1080/07448481.2013.843533. }\end{array}$ \\
\hline Art. 9 & $\begin{array}{l}\text { Macaskill, A. (2018). Undergraduate mental health issues: The challenge of the second year of study. Journal of } \\
\text { mental health (Abingdon, England), } 27(3), 214-221 \text {. doi: } 10.1080 / 09638237.2018 .1437611\end{array}$ \\
\hline Art. 10 & $\begin{array}{l}\text { Moreno, P. F.; Soares, A. B. (2014). O que vai acontecer quando eu estiver na universidade? Expectativas de jovens } \\
\text { estudantes brasileiros. Aletheia, (45), 114-127. Recuperado de http://pepsic.bvsalud.org/scielo.php?script=sci_ } \\
\text { arttext\&pid=S1413-03942014000200009\&Ing=pt\&tlng=pt. }\end{array}$ \\
\hline Art. 11 & $\begin{array}{l}\text { Oliveira, C. T.; Dias, A. C. G. (2014). Dificuldades na trajetória universitária e rede de apoio de calouros e formandos. } \\
\text { Psico, 45(2), 187-97. doi https://doi.org/10.15448/1980-8623.2014.2.13347 }\end{array}$ \\
\hline Art. 12 & $\begin{array}{l}\text { Oliveira, C. T.; Dias, A. C. G.; Piccoloto, N. M. (2013). Contributions of cognitive behavioral therapy for difficulties in } \\
\text { college adjustment. Revista Brasileira de Terapias Cognitivas, 9(1), 10-18. doi:10.5935/1808-5687.20130003. }\end{array}$ \\
\hline
\end{tabular}




\begin{tabular}{|c|c|}
\hline Código & Referência completa \\
\hline Art. 13 & $\begin{array}{l}\text { Oliveira, C. T. de; Santos, A. S. dos; Dias, A. C. G. (2016). Expectativas de universitários sobre a universidade: sugestões para } \\
\text { facilitar a adaptação acadêmica. Revista Brasileira de Orientação Profissional, 17(1), 43-53. Recuperado de http://pepsic. } \\
\text { bvsalud.org/scielo.php?script=sci_arttext\&pid=S1679-33902016000100006\&lng=pt\&tlng=pt. }\end{array}$ \\
\hline Art. 14 & $\begin{array}{l}\text { Polydoro, S. A. J.; Primi, R.; Serpa, M. N. F.; Zaroni, M. M. H.; Pombal, K. C. P. (2001). Desenvolvimento de uma escala } \\
\text { de integração ao ensino superior. Psico-USF, 6(1), 11-17. doi:10.1590/S1413-82712001000100003. }\end{array}$ \\
\hline Art. 15 & $\begin{array}{l}\text { Ridner, S. L.; Newton, K. S.; Staten, R. R.; Crawford, T. N.; Hall, L. A. (2016). Predictors of well-being among college } \\
\text { students. Journal of American College Health, 64(2), 116-124. doi:10.1080/07448481.2015.1085057. }\end{array}$ \\
\hline Art. 16 & $\begin{array}{l}\text { Sarriera, J. C.; Paradiso, A. C.; Schütz, F. F.; Howes, G. P. (2012). Estudo comparativo da integração ao contexto } \\
\text { universitário entre estudantes de diferentes instituições. Revista Brasileira de Orientação Profissional, 13(2), 163- } \\
\text { 172. Recuperado de http://pepsic.bvsalud.org/scielo.php?script=sci_arttext\&pid=S16793902012000200004\&lng= }\end{array}$ \\
\hline Art. 17 & $\begin{array}{l}\text { Schweitzer, R. D. (1996). Problems and awareness of support services among students at an urban Australian } \\
\text { university. Journal of American College Health, 45(2), 73-77. doi:10.1080/07448481.1996.9936865. }\end{array}$ \\
\hline Art. 18 & $\begin{array}{l}\text { Soares, A. B.; Francischetto, V.; Dutra, B. M.; Miranda, J. M.; Nogueira, C. C. C.; Leme, V. R.; Araújo, A.M.; Almeida, } \\
\text { L. S. (2014). O impacto das expectativas na adaptação acadêmica dos estudantes no Ensino Superior. Psico-USF, } \\
\text { 19(1), 49-60. doi:10.1590/S1413-82712014000100006 }\end{array}$ \\
\hline Art. 19 & $\begin{array}{l}\text { Stoliker, B. E.; Lafreniere, K. D. (2015). The influence of perceived stress, loneliness, and learning burnout on } \\
\text { university students' educational experience. College Student Journal, 49(1), 146-160. }\end{array}$ \\
\hline Art. 20 & $\begin{array}{l}\text { Teixeira, M. A. P.; Castro, A. K. S. S.; Zoltowski, A. P. C. (2012). Integração acadêmica e integração social nas } \\
\text { primeiras semanas na universidade: percepções de estudantes universitários. Gerais: Revista Interinstitucional } \\
\text { de Psicologia, 5(1), 69-85. Recuperado de:http://pepsic.bvsalud.org/scielo.php?script=sci_arttext\&pid=S1983- } \\
82202012000100006 \& \operatorname{lng}=\text { pt\&tIng=pt. }\end{array}$ \\
\hline Art. 21 & $\begin{array}{l}\text { Teixeira, M. A. P.; Castro, G. D.; Piccolo, L. D. R. (2007). Adaptação à universidade em estudantes universitários: Um } \\
\text { estudo correlacional. Interação em Psicologia, 11(2), 211-220. doi:10.5380/psi.v11i2.7466. }\end{array}$ \\
\hline Art. 22 & $\begin{array}{l}\text { Teixeira, M. A. P.; Dias, A. C. G.; Wottrich, S. H.; Oliveira, A. M. (2008). Adaptação à universidade em jovens calouros. } \\
\text { Psicologia Escolar e Educacional, 12(1), 185-202. doi:10.1590/S1413-85572008000100013. }\end{array}$ \\
\hline Art. 23 & $\begin{array}{l}\text { Withey, L.; Fox, C. L.; Hartley, J. (2014). 'I cannot mess this up anymore': The experiences of undergraduates who } \\
\text { withdraw and start again elsewhere. Psychology Teaching Review, 20(1), 78-89. }\end{array}$ \\
\hline
\end{tabular}

Fonte: Elaboração própria.

contendo as seguintes variáveis: (1) características gerais dos estudos (número do artigo - ID, Autores, Objetivos, Instrumentos, Variáveis investigadas, Delineamento, Participantes - Quantidade, idade e ano do curso, Curso/ área e Instituição) e (2) variáveis relativas à adaptação: dificultadores para a adaptação, consequências da não adaptação, facilitadores da adaptação, sintomas, repertório requerido à adaptação e estratégias de ensino. Para armazenamento e leitura dos textos selecionados foi utilizado o software de gerenciamento bibliográfico Mendeley.

\section{PROCEDIMENTO DE COLETA, REGISTRO E ANÁLISE DE DADOS}

Os artigos selecionados foram lidos na íntegra, sendo grifados os trechos que continham informações sobre as seguintes categorias estabelecidas a priori: dificultadores para a adaptação (e.g., características do ensino superior; nível de exigência), facilitadores da adaptação (e.g., rede de apoio; fornecimento de informações), consequências da não adaptação (e. g., baixo comprometimento do aluno; baixo desempenho acadêmico), sintomas (e. g., estresse, ansiedade depressão), repertório dos estudantes requerido à adaptação (e. g., resiliência; auto eficácia; autonomia; coping) e estratégias de ensino desse repertório (e. g., treino de resiliência; workshop de gestão do tempo). Essas categorias foram estabelecidas a partir da leitura dos textos mais citados nos artigos recuperados (Art. 6, Art. 21, Art. 22) e foram criadas por nomearem as variáveis mais citadas na investigação da adaptação à universidade. Para registro dos dados relativos às variáveis da adaptação, a pesquisadora lia o texto integralmente e grifava os trechos que continham informações sobre variáveis relativas às categorias pré-estabelecidas.

A partir do trecho destacado conforme a categoria a priori à qual se referia, foram criadas subcategorias com base na natureza da informação indicada naquele trecho. A subcategoria era transcrita para a tabela de acordo com a coluna relativa à categoria representada, na linha respectiva ao artigo que estava sendo categorizado. Por exemplo, no trecho: " $E$, ao ingressar na universidade, talvez fosse importante um projeto de integração dos calouros que abordasse com eficácia os serviços fundamentais já oferecidos por ela", foi identificada a subcategoria "fornecimento de informações", que foi transcrita para a tabela na coluna "Facilitadores da adaptação". Por fim, foi feita uma revisão das variáveis nomeadas a fim de agrupar aquelas 
que se referiam a um mesmo aspecto (e.g., acolhimento dos estudantes e atividades de integração - se referem a "rede de apoio"). Para controle desse agrupamento das variáveis, foi criada uma tabela em editor de texto com o nome das subcategorias e com as variáveis que foram agrupadas em cada uma delas.

A análise dos dados foi feita a partir das características gerais dos estudos e das variáveis da adaptação ao contexto acadêmico. Para isso, foram criadas tabelas com o nome de cada categoria, seguida do agrupamento das variáveis referentes àquela categoria, assim como a frequência absoluta e relativa com as quais foram citadas nos estudos.

\section{RESULTADOS E DISCUSSÃO}

\section{Características gerais dos estudos}

A partir da análise dos estudos, observa-se que a maioria foi publicada nos anos de 2014, 2016 e 2018. Do total de artigos analisados, apenas um foi publicado antes dos anos 2000 (Art. 17) ${ }^{1}$. Houve três gaps de publicação: de 1997 a 2000, de 2002 a 2006 e 2009 a 2010. A partir de 2010, foram encontradas publicações em todos os anos seguintes, até 2019. Dos 23 artigos analisados, 12 foram publicados internacionalmente e

${ }^{1}$ As referências dos artigos aqui citados foram apresentadas na Tabela 1.
11 no Brasil. Os estudos internacionais se dividem em Estados Unidos (13\%), Austrália (8,7\%), Reino Unido $(8,7 \%)$ Canadá (4,3\%), Espanha (4,3\%), Etiópia (4,3\%) e Singapura (4,3\%). Dos estudos internacionais, quatro foram publicados no Journal of American College Health (Art. 5, Art. 8, Art. 15 e Art. 17). A maior parte dos estudos foi realizada com estudantes de universidade pública (Art. 1, Art. 4, Art. 11, Art. 12, Art. 13, Art. 16, Art. 18, Art. 19, Art. 20, Art. 21, Art. 22, Art. 23), sendo que dois desses também incluíram estudantes de universidades particulares (Art. 16 e Art. 18). Os dados sobre as características dos participantes, instrumentos e delineamento dos estudos estão descritos na Tabela 2.

Com relação ao ano de graduação, em 14 estudos foram selecionados estudantes do primeiro ano. Com exceção de três estudos que selecionaram apenas estudantes de Psicologia, de modo geral, os estudos procuraram abranger uma diversidade de cursos na amostra. $O$ curso mais frequente foi o de Psicologia (43\%), seguido de Engenharia (26\%), Direito (17\%), Enfermagem (17\%), Administração (8,7\%), Economia $(8,7 \%)$ e Veterinária (8,7\%). Do ponto de vista metodológico, a maioria das pesquisas examinadas é descritiva (78\%), i. e., descreve as características de determinada população ou fenômeno e estabelece relações entre variáveis (Gil, 1999), no caso, a adaptação do estudante ao ensino superior e sua relação com a saúde mental. Os artigos analisados contribuem com

Tabela 2 - Características Dos Artigos Analisados Quanto Ao Curso (Ano), Participantes, Instituição E Delineamento Utilizado.

\begin{tabular}{|c|c|c|c|c|c|}
\hline \multirow{2}{*}{$\begin{array}{l}\text { Cód. do } \\
\text { Artigo }\end{array}$} & \multirow{2}{*}{ Curso (ano) } & \multicolumn{2}{|c|}{ Participantes } & \multirow{2}{*}{ Instrumentos } & \multirow{2}{*}{ Delineamento } \\
\hline & & Qtd. & Idade & & \\
\hline Art. 1 & N. E (primeiro ano) & 633 & N.E & $\begin{array}{l}\text { - Student Adaptation to College } \\
\text { Questionnaire (SACQ) }\end{array}$ & $\begin{array}{l}\text { Descritivo, transversal } \\
\text { e correlacional }\end{array}$ \\
\hline Art. 2 & $\begin{array}{l}\text { Psicologia, Engenharia Ambiental } \\
\text { e Engenharia de Produção, Enfer- } \\
\text { magem, Fisioterapia, Engenharia } \\
\text { de Alimentos, Medicina, Enge- } \\
\text { nharia Elétrica, Física e Nutrição } \\
\text { (primeiro ao quarto ano) }\end{array}$ & 22 & $18-25$ & $\begin{array}{l}\text { - Questionário de Ajustamento Acadêmico } \\
\text { - Escala de Adaptabilidade de Carreira }\end{array}$ & $\begin{array}{l}\text { Pesquisa-ação com } \\
\text { delineamento quase- } \\
\text { experimental }\end{array}$ \\
\hline Art. 3 & N.E (primeiro e último ano) & 150 & $M=23,2$ & $\begin{array}{l}\text { - Kessler Psychological Distress Scale (K10) } \\
\text { - General Health Questionnaire (GHQ-12) } \\
\text { - Brief Symptom Inventory (BSI) } \\
\text { - Warwick Edinburgh Mental Well Being } \\
\text { Scale (WEMWBS) } \\
\text { - Brief Resilience Scale (BRS) }\end{array}$ & $\begin{array}{l}\text { Descritivo, transversal } \\
\text { e correlacional }\end{array}$ \\
\hline Art. 4 & $\begin{array}{l}\text { Ciências da Saúde, Ciências, En- } \\
\text { genharia, Ciências Sociais, Artes e } \\
\text { Humanidades (segundo ano) }\end{array}$ & 706 & $M=20,2$ & $\begin{array}{l}\text { - Evaluation of the Need for Psychological } \\
\text { Assistance } \\
\text { Questionnaire (NPAQ) } \\
\text { - Hospital Anxiety and Depression Scale } \\
\text { (HADS) }\end{array}$ & $\begin{array}{l}\text { Descritivo, transversal } \\
\text { e correlacional }\end{array}$ \\
\hline Art. 5 & $\begin{array}{l}\text { Artes, Biologia, Ciências, Comu- } \\
\text { nicação, Educação, Jornalismo, } \\
\text { Psicologia (primeiro e último ano) }\end{array}$ & 605 & $M=21,03$ & $\begin{array}{l}\text { - Variables Related to Academic Persistence } \\
\text { and Demographics } \\
\text { - Sen se of B e long ing (SOB) } \\
\text { - Connor-Davidson Resilience Scale (CD-RISC) } \\
\text { - Social Support Questionnaire (SSQ-6) } \\
\text { - Mental Health Inventory-5 (MHI-5) }\end{array}$ & $\begin{array}{l}\text { Descritivo, transversal } \\
\text { e correlacional }\end{array}$ \\
\hline
\end{tabular}




\begin{tabular}{|c|c|c|c|c|c|}
\hline \multirow{2}{*}{$\begin{array}{l}\text { Cód. do } \\
\text { Artigo }\end{array}$} & \multirow{2}{*}{ Curso (ano) } & \multicolumn{2}{|c|}{ Participantes } & \multirow{2}{*}{ Instrumentos } & \multirow{2}{*}{ Delineamento } \\
\hline & & Qtd. & Idade & & \\
\hline Art. 6 & $\begin{array}{l}\text { Psicologia (segundo, terceiro e } \\
\text { quarto ano) }\end{array}$ & 203 & $M=20,17$ & $\begin{array}{l}\text { - Questionário de Vivências Acadêmicas - } \\
\text { Versão Reduzida (QVA-r) } \\
\text { - Questão sobre as expectativas no ingresso }\end{array}$ & $\begin{array}{l}\text { Descritivo, transversal } \\
\text { e correlacional }\end{array}$ \\
\hline Art. 7 & $\begin{array}{l}\text { Administração, Artes } \\
\text { Ciências, Enfermagem, Medicina, } \\
\text { Saúde (primeiro ano) }\end{array}$ & 630 & $M=19,56$ & $\begin{array}{l}\text { - Psychological Well-Being Scale (PWB) } \\
\text { - The mindful attention awareness scale (MAAS) } \\
\text { - The Generalized Self-Efficacy Scale (GSES) } \\
\text { - The 12-item multi-dimensional scale of } \\
\text { perceived social support (MSS) }\end{array}$ & $\begin{array}{l}\text { Descritivo, transversal } \\
\text { e correlacional }\end{array}$ \\
\hline Art. 8 & N.E (primeiro ano) & 76 & $M=18,37$ & $\begin{array}{l}\text { - System Usability Scale (SUS) } \\
\text { - ACT Knowledge Questionnaire } \\
\text { - Depression, Anxiety and Stress Scale (DASS) } \\
\text { - Acceptance and Action Questionnaire-II (AAQ-II) } \\
\text { - Personal Values Questionnaire (PVQ) }\end{array}$ & $\begin{array}{l}\text { Experimental de gru- } \\
\text { po com pré e pós tes- } \\
\text { te e follow up }\end{array}$ \\
\hline Art. 9 & $\begin{array}{l}\text { Criminologia, Direito, Geografia, In- } \\
\text { glês, Educação, Sociologia, Política } \\
\text { e Psicologia (segundo ano) }\end{array}$ & 23 & $M=20,81$ & $\begin{array}{l}\text { - Entrevista } \\
\text { - General Health Questionnaire-28 (GHQ- 28) }\end{array}$ & Descritivo qualitativo \\
\hline Art. 10 & $\begin{array}{l}\text { Administração, Biomedicina, En- } \\
\text { genharia, Enfermagem, Estatística, } \\
\text { História, Matemática, Psicologia } \\
\text { (primeiro e último ano) }\end{array}$ & 13 & $M=19,54$ & - Entrevista semiestruturada & Descritivo qualitativo \\
\hline Art. 11 & Economia, Psicologia (N.E) & 24 & $17-26$ & - Entrevista semiestruturada & Descritivo qualitativo \\
\hline Art. 12 & $\begin{array}{l}\text { Administração, Agronegócio, Artes } \\
\text { Cênicas, Ciências Biológicas, Ciên- } \\
\text { cias Contábeis, Educação Especial, } \\
\text { Enfermagem, Fonoaudiologia, } \\
\text { Matemática, Medicina Veterinária, } \\
\text { Sistemas de Informação, Superior } \\
\text { em Produção de Grãos (primeiro, } \\
\text { penúltimo e último ano) }\end{array}$ & 504 & $M=23,5$ & $\begin{array}{l}\text { - Questão aberta sobre as principais dificul- } \\
\text { dades encontradas no contexto acadêmico } \\
\text { durante o primeiro ano da graduação }\end{array}$ & Descritivo qualitativo \\
\hline Art. 13 & Economia, Psicologia (N.E) & 20 & $M=21,95$ & Entrevista semiestruturada & Descritivo qualitativo \\
\hline Art. 14 & $\begin{array}{l}\text { Análise de Sistemas, Eng. da Com- } \\
\text { putação, Farmácia, Letras, Meca- } \\
\text { trônica, Odontologia, } \\
\text { Psicologia (N.E) }\end{array}$ & 633 & N.E & $\begin{array}{l}\text { - Escala de Integração ao Ensino Superior } \\
\text { (EIES) }\end{array}$ & Correlacional \\
\hline Art. 15 & N.E (N.E) & 568 & $M=20,4$ & $\begin{array}{l}\text { - The National College Health Assessment } \\
\text { (NCHA II) } \\
\text { - The Public Health Surveillance Well- } \\
\text {-Being Scale (PHS-WB) }\end{array}$ & $\begin{array}{l}\text { Descritivo, transversal } \\
\text { e correlacional }\end{array}$ \\
\hline Art. 16 & Psicologia (N.E) & 273 & $M=23,89$ & $\begin{array}{l}\text { - Questionário de Vivências Acadêmicas - } \\
\text { Versão Reduzida (QVA-r) }\end{array}$ & $\begin{array}{l}\text { Descritivo, transversal } \\
\text { e correlacional }\end{array}$ \\
\hline Art. 17 & $\begin{array}{l}\text { Direito, Engenharia, Humanas } \\
\text { (primeiro ano) }\end{array}$ & 441 & N.E & - Questionário desenvolvido para o estudo & $\begin{array}{l}\text { Descritivo, transversal } \\
\text { e correlacional }\end{array}$ \\
\hline Art. 18 & $\begin{array}{l}\text { Medicina, Outros, Pedagogia, Psi- } \\
\text { cologia (todos os anos) }\end{array}$ & 182 & $M=26,2$ & $\begin{array}{l}\text { - Questionário de Vivências Acadêmicas - } \\
\text { versão reduzida (QVA-r) } \\
\text { - Questionário de Envolvimento Acadê- } \\
\text { mico (QEA) } \\
\text { - Questionário de Classificação Econômi- } \\
\text { ca do Brasil }\end{array}$ & $\begin{array}{l}\text { Descritivo, transversal } \\
\text { e correlacional }\end{array}$ \\
\hline Art. 19 & Psicologia (primeiro ano) & 150 & $M=21$ & $\begin{array}{l}\text { - Perceived Stress Scale (PSS) } \\
\text { - Academic Coping Strategies Scale (ACSS) } \\
\text { - Maslach Burnout Inventory-Student } \\
\text { Survey (MBI-SS) } \\
\text { - Utrecht Work Engagement Scale for } \\
\text { Students (UWES-S) } \\
\text { - The University of California, Los Angeles } \\
\text { Loneliness Scale-Version } 3 \text { (UCLA-3) }\end{array}$ & $\begin{array}{l}\text { Descritivo, transversal } \\
\text { e correlacional }\end{array}$ \\
\hline Art. 20 & $\begin{array}{l}\text { Enfermagem, Engenharia Civil, Far- } \\
\text { mácia, Jornalismo (todos os anos) }\end{array}$ & 17 & $18-28$ & - Entrevista semiestruturada & $\begin{array}{l}\text { Qualitativo e explora- } \\
\text { tório com estudo de } \\
\text { caso coletivo }\end{array}$ \\
\hline
\end{tabular}




\begin{tabular}{|c|c|c|c|c|c|}
\hline \multirow{2}{*}{$\begin{array}{l}\text { Cód. do } \\
\text { Artigo }\end{array}$} & \multirow{2}{*}{ Curso (ano) } & \multicolumn{2}{|c|}{ Participantes } & \multirow{2}{*}{ Instrumentos } & \multirow{2}{*}{ Delineamento } \\
\hline & & Qtd. & Idade & & \\
\hline Art. 21 & $\begin{array}{l}\text { Direito, Psicologia, Veterinária } \\
\text { (primeiro ano) }\end{array}$ & 342 & $M=21,2$ & $\begin{array}{l}\text { - Questionário de Vivências Acadêmicas - } \\
\text { versão reduzida (QVA-r) } \\
\text { - Nível de participação em atividades } \\
\text { extracurriculares } \\
\text { - Escalas Likert desenvolvidas para o estudo }\end{array}$ & $\begin{array}{l}\text { Descritivo, transversal } \\
\text { e correlacional }\end{array}$ \\
\hline Art. 22 & $\begin{array}{l}\text { Administração, Agronomia, Direi- } \\
\text { to, Educação especial, Educação } \\
\text { física, Engenharia Civil, Engenha- } \\
\text { ria elétrica, Engenharia mecânica, } \\
\text { Matemática, Química (N.E) }\end{array}$ & 14 & $18-22$ & - Entrevista semiestruturada & $\begin{array}{l}\text { Descritivo, qualitativo } \\
\text { fenomenológico }\end{array}$ \\
\hline Art. 23 & $\begin{array}{l}\text { Ciências Sociais, Inglês, Música, } \\
\text { Política (primeiro e último ano) }\end{array}$ & 5 & $18-21$ & - Entrevista semiestruturada & Descritivo qualitativo \\
\hline
\end{tabular}

Fonte: Elaboração própria.

a descrição do fenômeno, permitindo o conhecimento da realidade dos estudantes e análises correlacionais de variáveis que podem influenciar na sua adaptação ao ensino superior.

Essas análises são importantes para indicar aspectos a serem investigados empiricamente, mas por si só ainda são insuficientes, visto que tratam de correlações e não de relações de causalidade. Apesar da importância desse tipo de pesquisa, há necessidade de desenvolver também estudos experimentais e aplicados, pois à medida que as variáveis relacionadas a esse fenômeno são mais conhecidas, é preciso investigar também de que forma é possível intervir sobre ele. Com relação a intervenções com essa população, foram encontrados apenas dois estudos (Art. 2 e Art. 8), que apesar de apresentarem o desenvolvimento de uma intervenção e avaliação dos seus resultados, não especificam os comportamentos que foram ensinados aos estudantes. Essa especificação poderia auxiliar no desenvolvimento de treinamentos e outros tipos de intervenção, uma vez que produz visibilidade acerca do que os estudantes necessitam ser capazes de fazer (competências, habilidades a desenvolver) (Santos, Kienen, Viecili, Botomé, \& Kubo, 2009) para lidar com o contexto universitário de modo a terem um bom desempenho acadêmico e manterem-se saudáveis física e psicologicamente.

Dos instrumentos aplicados, destaca-se o Questionário de Vivências Acadêmicas - Versão Reduzida (QVA-r), que foi utilizado em quatro dos estudos analisados. Todos os estudos quantitativos utilizaram pelo menos dois instrumentos, e todos objetivaram fazer correlações entre variáveis, tanto sociodemográficas quanto variáveis específicas, como níveis de ansiedade, depressão e variáveis acadêmicas. Com relação aos participantes, houve grande variedade de estudantes que participaram dos estudos, sendo maior nos estudos quantitativos. A média de idade também variou, mas manteve-se entre 18 e 26,2 anos.

\section{Variáveis da adaptação ao contexto acadêmico}

A identificação das variáveis específicas presentes no contexto do ensino superior permite uma compreensão maior de quais aspectos vivenciados pelos estudantes podem estar relacionados à sua adaptação a esse contexto e sua saúde, possibilitando o desenvolvimento de intervenções mais diretas e eficazes. Na Tabela 3 estão descritos os principais facilitadores e dificultadores da adaptação, assim como as consequências da não adaptação ao ensino superior.

Fatores relacionados à rede de apoio $(47,8 \%)$, fornecimento de informações $(39,1 \%)$ e integração acadêmica $(34,8 \%)$ apareceram com maior frequência como facilitadores da adaptação ao ensino superior, seguidos de características da instituição relativas à infraestrutura $(26,1 \%)$ e integração social $(26,1 \%)$. Esses dados indicam a importância e viabilidade da realização de intervenções em variáveis ambientais, assim como a responsabilidade de gestores da universidade e professores sobre isso. Dentre as variáveis ambientais, disponibilizar informações e serviços de apoio aos ingressantes na universidade pode contribuir para a adaptação, de modo a garantir o acesso às oportunidades oferecidas pela instituição (Art. 6; Art. 11; Art. 16). Isso porque os estudantes, em sua maioria, ingressam no ensino superior se sentindo "perdidos" e sem saber onde buscar ajuda, esclarecer dúvidas, ou até mesmo desconhecendo a existência dos serviços de apoio da instituição.

Além disso, os docentes podem contribuir para a adaptação dos estudantes ao se mostrarem disponíveis para esclarecerem dúvidas e para receberem ideias e sugestões dos alunos (Art. 13), portanto, a capacitação dos docentes é outro fator relevante para a adaptação acadêmica. Considerando que a saúde mental do estudante universitário está relacionada a sua adaptação a esse contexto, ter uma rede de apoio e integrar-se socialmente no ambiente universitário é de extrema importância para que os estudantes conversem sobre as dificuldades que estão enfrentando, compartilhem experiências, saibam onde buscar ajuda quando precisarem e que se sintam 
acolhidos nesse novo contexto, caracterizado por várias situações novas e desafiadoras para muitos estudantes, principalmente nos primeiros anos do curso (Macaskill, 2018; Oliveira \& Dias, 2014).

Outro aspecto importante é que os estudantes sejam estimulados a realizar atividades extracurriculares (Art. 10; Art. 18; Art. 20), pois isso permite um maior contato com a prática profissional e a exploração da carreira desde o início da graduação, motivando-os a continuarem os estudos e a estabelecerem relações entre a teoria estudada e a prática. Explorar as oportunidades do ambiente acadêmico envolve a busca de informações sobre a realidade do trabalho e sobre a área de conhecimento, possibilitando uma maior clareza acerca das alternativas educacionais e profissionais existentes para o estudante (Teixeira, Bardagi, \& Hutz, 2007). Essa exploração é importante porque, muitas vezes, os cursos não abordam todas as possibilidades de atuação profissional existentes no mundo do trabalho, o que pode frustrar as expectativas iniciais dos estudantes e dificultar a sua adaptação.

Quanto aos fatores dificultadores da adaptação ao ensino superior, foram descritos tanto aspectos pessoais quanto específicos da vida acadêmica. Fatores pessoais como relacionamentos interpessoais (56,5\%), saída de casa $(56,5 \%)$, falta de rede de apoio (52,2\%) e situação financeira $(43,5 \%)$ foram identificados com maior frequência nos artigos analisados. Com relação a aspectos específicos da vida acadêmica, se destacaram o nível de exigência $(73,9 \%)$ e as características do Ensino Superior (39,1\%). Esses dificultadores parecem exigir uma capacidade de resolução de problemas por parte dos universitários, visto que esse novo contexto, tanto o ingresso na universidade quanto a possível saída de casa apresentam situações novas para os estudantes que geralmente trazem problemas com os quais eles precisam aprender a lidar e a resolver sozinhos. A resolução de problemas se caracteriza como um processo comportamental complexo que envolve não só a alteração do ambiente, mas também do próprio indivíduo e da situação, de modo que a ocorrência de uma resposta solução seja favorecida (Skinner, 1974). Aprender a manejar aspectos do ambiente para tornar a solução de um problema mais provável parece ser um repertório importante para os estudantes, visto que eles poderão se deparar a situações novas e complexas para as quais não terão uma resposta pronta à disposição, requerendo respostas prévias para chegarem a uma solução.

Uma dessas respostas prévias pode estar no âmbito dos relacionamentos interpessoais, uma vez que pedir ajuda, tirar dúvidas e buscar informações pode aumentar a probabilidade de solução de um problema. Para isso, o estudante precisa se relacionar com pessoas diferentes, e com autoridades como professores, gestores, funcionários, repertório que pode não ter sido desenvolvido previamente enquanto frequentava outros níveis de ensino. É necessário desenvolver comportamentos relacionados às habilidades sociais, repertório que implica na capacidade de iniciar e manter conversas, falar em grupo, expressar sentimentos, defender os próprios direitos, pedir ajuda, recusar pedidos, entre outros (Caballo, 1996; Del Prette \& Del Prette, 2009). Isso poderia auxiliar os estudantes a conseguirem se expressar com relação à insatisfação com notas ou correções, dúvidas quanto ao funcionamento da instituição, questionamentos sobre a ementa ou formas de avaliação, entre outros.

No que diz respeito às consequências da não adaptação ao ensino superior, as principais categorias identificadas foram abandono do curso $(52,2 \%)$ e baixo desempenho acadêmico (34,8\%), seguidas de baixo comprometimento (13\%) e saúde mental e física (13\%). A problemática do abandono do ensino superior parece estar diretamente relacionada com as dificuldades de adaptação do estudante à universidade (Moreno \& Soares, 2014; Polydoro et al., 2001; Withey, Fox, \& Hartley, 2014). A identificação e sistematização das principais variáveis que dificultam a adaptação do estudante e, consequentemente, sua permanência na universidade, possibilita o desenvolvimento de ações no ambiente universitário que modifiquem esses dificultadores ou que deem condições para que os estudantes lidem com as situações-problema de uma forma mais saudável e produtiva.

Além da descrição dos problemas que ocorrem ao ingressar no ensino superior, os artigos também contêm informações acerca dos sintomas apresentados pelos estudantes nesse contexto e dos fatores e comportamentos desses estudantes que facilitam a adaptação, como apresentado na Tabela 4.

Os principais sintomas apresentados por estudantes universitários foram ansiedade (54,2\%), estresse $(52,2 \%)$ e depressão (52,2\%). Esses sintomas, em uma visão analítico-comportamental, são compreendidos como um conjunto complexo de comportamentos que ocorrem devido a um padrão de interação de um indivíduo com o seu ambiente (Cavalcante, 1997). Algumas contingências ambientais comumente produzem padrões emocionais, os "sintomas". No contexto universitário, alguns fatores específicos que dificultam a adaptação podem contribuir para o desenvolvimento desses sintomas, como o nível de exigência do curso (Art. 10), infraestrutura da instituição (Art. 13) e relacionamentos interpessoais (Art. 9). A identificação desses fatores parece indicar que os estudantes não são intrinsecamente ansiosos ou estressados, mas que alguns aspectos da interação deles com o ambiente universitário estão maximizando esses sintomas. Ao identificar esses aspectos, é possível desenvolver estratégias para modificar essa interação e, consequentemente, minimizar os sintomas apresentados. Além do desenvolvimento desses sintomas, muitas vezes os dificultadores interferem na permanência ou abandono do curso, no próprio 
desempenho acadêmico, no comprometimento com as atividades acadêmicas, no desenvolvimento intelectual e pessoal do estudante e também na motivação para os estudos e para a construção da carreira profissional (Art. 8, Art. 13, Art. 18).

Para prevenção desses sintomas e do abandono do curso, é importante preparar melhor os estudantes para lidarem com as novas demandas desse contexto, a partir do desenvolvimento de repertórios específicos para isso. Ao ingressar no ensino superior, o estudante se depara com diversas situações novas, problemas que precisará resolver rotineiramente, relacionamentos interpessoais com colegas, professores, funcionários e gestores, além de um nível de exigência e sobrecarga de atividades com os quais não estava acostumado no ensino médio (Art. 6, Art. 11). Apesar de muitos desses fatores estarem fora do controle do estudante, é possível que ele aprenda a se comportar de modo a lidar com os dificultadores existentes nesse contexto e se adaptar ao ensino superior de modo mais satisfatório, diminuindo os prejuízos a sua saúde mental e consequências como abandono do curso, desmotivação, baixo desempenho acadêmico, entre outros (Art. 21; Art. 22). Para isso, é necessário que os estudantes aprendam a se comportar efetivamente frente às situações-problema desse contexto, e não apenas a "falar sobre" o que pode ser feito (Kubo \& Botomé, 2001), a partir de temas específicos, como resiliência, desenvolvimento pessoal e gestão do tempo (Art. 3, Art. 13). Saber definir o que é resiliência e que tipo de situações exigem que o estudante seja resiliente ainda não é suficiente para que ele se comporte de forma resiliente durante a sua vivência no ensino superior. Isso destaca novamente a importância de pesquisas que descrevam claramente os comportamentos que os estudantes necessitam desenvolver para manejar adequadamente tais situações.

Nos artigos analisados, destacaram-se repertórios de autonomia (43,5\%) e comprometimento $(34,8 \%)$, seguidos de comportamentos de estudo $(26,1 \%)$, manejo das emoções (26,1\%), autoeficácia (26,1\%) e coping (26,1\%). Aprender a gerir as atividades acadêmicas e pessoais e também desenvolver um repertório de estudo (Art. 3) parece ser importante para que os estudantes consigam lidar com as exigências e, ao mesmo tempo, conciliá-las com a vida pessoal, estabelecendo relacionamentos interpessoais mais satisfatórios e uma rede de apoio para compartilharem suas frustrações, dificuldades e experiências na universidade (Art. 7), fatores que parecem facilitar a adaptação (Tabela 3). Gerir as atividades acadêmicas e pessoais e desenvolver um repertório de estudo são comportamentos complexos e requeridos ao longo de toda a vida acadêmica e profissional, porém, raramente são alvo de ensino (Kienen et al., 2017; Yoshiy \& Kienen, 2018). Desenvolver intervenções que objetivem o ensino desses repertórios pode auxiliar os estudantes a aprender a estudar de modo mais eficiente,

Tabela 3 - Facilitadores e Dificultadores da Adaptação e Consequências da Não Adaptação.

\begin{tabular}{|c|c|}
\hline Facilitadores da adaptação & Artigos \% (N) \\
\hline Rede de apoio & $47,8(N=11)$ \\
\hline Fornecimento de informações & $39,1(N=9)$ \\
\hline Integração acadêmica & $34,8(N=8)$ \\
\hline Características da instituição - infraestrutura & $26,1(N=6)$ \\
\hline Integração social & $26,1(N=6)$ \\
\hline Atividades extracurriculares & $21,7(N=5)$ \\
\hline Questões pedagógicas & $21,7(\mathrm{~N}=5)$ \\
\hline Relacionamentos interpessoais & $17,4(\mathrm{~N}=4)$ \\
\hline Contato com a profissão & $13(N=3)$ \\
\hline Lazer/atividade física & $13(N=3)$ \\
\hline Características da instituição - outros & $8,7(N=2)$ \\
\hline Expectativas quanto à transição para o ES & $8,7(\mathrm{~N}=2)$ \\
\hline Dificultadores para a adaptação & Artigos \% (N) \\
\hline Nível de exigência & $73,9(\mathrm{~N}=17)$ \\
\hline Relacionamentos interpessoais & $56,5(N=13)$ \\
\hline Saída de casa & $56,5(N=13)$ \\
\hline Falta de rede de apoio & $52,2(N=12)$ \\
\hline Situação financeira & $43,5(N=10)$ \\
\hline Características individuais - cognitivas e de personalidade & $39,1(N=9)$ \\
\hline
\end{tabular}


Características do ES

Falta de fornecimento de informações

Expectativas quanto à transição ao ES

Desempenho acadêmico

Gestão do tempo

Características da instituição - currículo

Características da instituição - questões burocráticas

Questões pedagógicas

Repertório de manejo de emoções

Repertório de estudo

Sobrecarga de atividades

Características da instituição - infraestrutura

Características individuais - condições de saúde

Caracteristicas individuais - condições de saúde

Novas responsabilidades

$$
\begin{aligned}
& 39,1(\mathrm{~N}=9) \\
& 34,8(\mathrm{~N}=8) \\
& 30,4(\mathrm{~N}=7) \\
& 30,4(\mathrm{~N}=7) \\
& 26,1(\mathrm{~N}=6) \\
& 26,1(\mathrm{~N}=6) \\
& 21,7(\mathrm{~N}=5) \\
& 17,4(\mathrm{~N}=4) \\
& 17,4(\mathrm{~N}=4) \\
& 17,4(\mathrm{~N}=4) \\
& 13(\mathrm{~N}=3) \\
& 13(\mathrm{~N}=3) \\
& 13(\mathrm{~N}=3) \\
& 4,3(\mathrm{~N}=1)
\end{aligned}
$$

Artigos \% (N)

Abandono do curso

$52,2(\mathrm{~N}=12)$

Baixo desempenho acadêmico $34,8(\mathrm{~N}=8)$

Baixo comprometimento $13(\mathrm{~N}=3)$

Saúde mental e física $13(\mathrm{~N}=3)$

Baixo desenvolvimento intelectual

$8,7(\mathrm{~N}=2)$

Baixo desenvolvimento pessoal

$8,7(\mathrm{~N}=2)$

Baixa motivação

$8,7(\mathrm{~N}=2)$

Baixa autoeficácia profissional

$8,7(\mathrm{~N}=2)$

Baixa autoconfiança

$4,3(\mathrm{~N}=1)$

Dificuldades de progresso na carreira

$4,3(\mathrm{~N}=1)$

4,3 $(\mathrm{N}=1)$

$4,3(\mathrm{~N}=1)$

Frustrações

$4,3(\mathrm{~N}=1)$

Qualidade das relações interpessoais

Fonte: Elaboração própria.

identificando quais são suas principais dificuldades e criando estratégias para lidar com elas, além de planejar as atividades de modo a incluir no seu planejamento atividades de lazer e de interação social.

Outro repertório relevante a ser desenvolvido pelos estudantes universitários é o de coping, especialmente porque o ambiente acadêmico, dada sua complexidade, pode envolver uma série de estressores com os quais os estudantes terão que lidar em seu cotidiano. O coping se refere aos recursos emocionais que o indivíduo utiliza para lidar com as situações estressoras (Lazarus \& Folkman, 1984). Os estudantes lidam de maneiras diferentes com os estressores, podendo utilizar métodos positivos, como aqueles relacionados à resolução de problemas e busca por suporte social ou atividades de lazer, ou estratégias não adaptativas, como a esquiva dos eventos estressores (Deasy, Coughlan, Pironom, Jourdan, \& Mannix-McNamara, 2014). Desenvolver intervenções relacionadas ao coping focado no problema e suporte social pode auxiliar os estudantes a manejarem as situações estressantes que vivenciam de forma a favorecer sua adaptação ao contexto universitário, reduzindo, minimizando ou tolerando o estresse gerado por essas situações (Carlotto, Teixeira, \& Dias, 2015).

No que diz respeito às estratégias de ensino utilizadas para desenvolver repertórios requeridos à adaptação, a estratégia mais frequente foi workshops de gestão do tempo (13\%), seguido de promover aumento dos recursos internos $(8,7 \%)$ e treino de resiliência (8,7\%). "Estratégias de Ensino" foi a categoria menos descrita nos artigos analisados, uma vez que apenas nove estudos (Art. 3; Art. 5; Art. 7; Art. 8; Art. 12; Art. 13; Art. 15; Art. 16; Art. 18) citaram estratégias que poderiam auxiliar professores ou gestores dos cursos a intervirem no processo de adaptação do estudante no ensino superior. Apesar de citarem algumas estratégias, não descrevem detalhadamente como elas poderiam ser aplicadas no contexto universitário, que condições deveriam garantir ou que comportamentos deveriam ser alvo de ensino, se limitando a temáticas (e. g., gestão do 
Tabela 4 - Sintomas Apresentados Pelos Estudantes e Repertório Requerido À Adaptação.

\begin{tabular}{|c|c|}
\hline Sintomas & Artigos \% (N) \\
\hline Ansiedade & $52,2(\mathrm{~N}=12)$ \\
\hline Estresse & $52,2(\mathrm{~N}=12)$ \\
\hline Depressão & $47,8(N=11)$ \\
\hline Doenças físicas & $4,3(N=1)$ \\
\hline Alterações de humor & $4,3(N=1)$ \\
\hline Dificuldade de concentração & $4,3(N=1)$ \\
\hline Distúrbios psicossomáticos & $4,3(N=1)$ \\
\hline Ideação suicida & $4,3(N=1)$ \\
\hline Problemas com o sono e/ou alimentação & $4,3(N=1)$ \\
\hline Repertório dos estudantes requeridos à adaptação & Artigos \% (N) \\
\hline Autonomia & $43,5(N=10)$ \\
\hline Comprometimento & $34,8(N=8)$ \\
\hline Comportamentos de estudo & $26,1(N=6)$ \\
\hline Manejo das emoções & $26,1(N=6)$ \\
\hline Autoeficácia & $26,1(N=6)$ \\
\hline Coping & $26,1(\mathrm{~N}=6)$ \\
\hline Ajustamento & $21,7(N=5)$ \\
\hline Comportamento exploratório vocacional & $21,7(N=5)$ \\
\hline Resiliência & $17,4(N=4)$ \\
\hline Autoconhecimento & $13(N=3)$ \\
\hline Responsabilidade & $13(N=3)$ \\
\hline Buscar ajuda & $8,7(N=2)$ \\
\hline Proatividade & $8,7(N=2)$ \\
\hline Criatividade & $4,3(\mathrm{~N}=1)$ \\
\hline Flexibilidade psicológica & $4,3(N=1)$ \\
\hline
\end{tabular}

Fonte: Elaboração própria.

tempo, resiliência) ou a teorias que poderiam subsidiar essas estratégias (e. g., Terapia Cognitivo-Comportamental, Terapia de Aceitação e Compromisso). É importante que essas estratégias sejam melhor operacionalizadas, para que outros profissionais que necessitem intervir com estudantes possam partir de dados sistematizados para elaborar suas próprias intervenções.

O contexto universitário é complexo e algumas de suas características podem maximizar o sofrimento dos estudantes. Isso pode ocorrer tanto pela falta de repertórios básicos para lidar com algumas situações, por acontecimentos externos que ocorrem durante o período da universidade ou por situações específicas que acontecem nessa etapa. É importante compreender que muitas dessas situações que podem ser estressoras para os estudantes na universidade, estão também presentes no mercado de trabalho. Isso indica a necessidade ainda maior de ensinar aos estudantes comportamentos para lidarem com essas situações, visto que eles continuarão se deparando com elas durante toda a sua vida. Intervenções realizadas no ambiente universitário (e.g., melhorar a infraestrutura da universidade, disponibilizar informações sobre processos burocráticos, aperfeiçoar o currículo de modo a incluir atividades práticas e relacionadas à profissão) podem favorecer a adaptação, porém não são suficientes para que os estudantes aprendam a lidar com as situações-problema que provavelmente surgirão.

\section{CONSIDERAÇÕES FINAIS}

Este estudo possibilitou sistematizar variáveis relacionadas às situações-problemas com as quais os estudantes se deparam no contexto universitário, assim como às consequências dessas para o estudante, seja com relação à motivação, permanência no curso ou para o desenvolvimento de sintomas como ansiedade, depressão e estresse. Possibilitou ainda identificar repertórios básicos requeridos dos estudantes para lidar com essas situações (e.g., autonomia, manejo de emoções). No entanto, tais repertórios ainda são descritos de forma genérica, requerendo mais 
especificação dos tipos de comportamentos que o estudante necessita desenvolver para poder se integrar à universidade de um modo mais saudável. Sugere-se então pesquisas que tenham como objetivo caracterizar tais comportamentos, assim como pesquisas de intervenção com essa população, que descrevam de forma detalhada os comportamentos que foram ensinados e também os resultados dessas intervenções.

Algumas limitações desse estudo devem ser consideradas. Primeiramente, como se propôs a compreender como os estudantes, de modo geral, se adaptam à universidade, vários estudos mais específicos foram excluídos da análise, como aqueles relacionados às dificuldades presentes em alguns cursos de graduação (e. g., cursos da área da saúde) e com populações diagnosticadas com algum transtorno específico (e. g., TDAH, transtornos de aprendizagem). Além disso, como foram analisados artigos nacionais e internacionais, não foi feita uma análise separada das variáveis culturais que podem influenciar na adaptação acadêmica.

Apesar dessas limitações, destaca-se que a sistematização das variáveis relativas à adaptação dos estudantes ao ensino superior e às consequências sobre a saúde mental deles indicam o papel do conhecimento psicológico, assim como das intervenções dele decorrentes, para a promoção do sucesso acadêmico e da formação integral dos estudantes como profissionais e cidadãos. Implementar ações de caráter preventivo, que visem ao bem estar físico e psicológico dos estudantes, bem como à adaptação saudável deles ao ensino superior, e que envolvam toda a comunidade universitária (e.g., estudantes, docentes, coordenadores de curso, colaboradores técnico administrativos) parecem ser uma alternativa que possibilita uma atuação crítica e reflexiva por parte dos psicólogos, além de ampliar as possibilidades de atuação profissional nesse contexto, tradicionalmente marcada por intervenções clínicas individuais voltadas ao público discente.

\section{REFERÊNCIAS}

Bore, M.; Pittolo, C.; Kirby, D.; Dluzewska, T.; Marlin, S. (2016). Predictors of psychological distress and well-being in a sample of Australian undergraduate students. Higher Education Research \& Development, 35(5), 869-880. doi: 10.1080/07294360.2016.1138452.

Caballo, V. E. (1996). O treinamento em habilidades sociais. In: V. E. Caballo (Ed.). Manual de técnicas de terapia e modificação do comportamento (pp. 3-42). São Paulo: Santos Livraria Editora.

Carlotto, R. C.; Teixeira, M. A. P.; Dias, A. C. G. (2015). Adaptação acadêmica e coping em estudantes universitários. PsicoUSF, 20(3), 421-432. doi: 10.1590/1413-82712015200305

Cavalcante, S. N. (1997). Notas sobre o fenômeno depressão a partir de uma perspectiva analítico-comportamental. Psicologia: Ciência e Profissão, 17(2), 2-12. doi:10.1590/ S1414-98931997000200002

Costa, A. B. C.; Zoltowski, A. P. C. (2014). Como escrever um artigo de revisão sistemática. In: S.H. Koller; M. C. P. de Paula Couto; J. Hohendorff (Eds.), Manual de produção científica (pp. 55-70). Porto Alegre: Penso.

Deasy, C.; Coughlan, B.; Pironom, J.; Jourdan, D.; MannixMcNamara, P. (2014). Psychological distress and coping amongst higher education students: A mixed method enquiry. PLOS ONE, 9(12), e115193. doi: 10.1371/journal. pone.0115193.

Del Prette, Z. A. P.; Del Prette, A. (2009). Avaliação de habilidades sociais: Bases conceituais, instrumentos e procedimentos. In A. Del Prette; Z. A. P. Del Prette (Eds.), Psicologia das habilidades sociais: Diversidade teórica e suas implicações (pp.187-229). Petrópolis: Vozes.

FONAPRACE (2014). IV Pesquisa do perfil do socioeconômico e cultural dos estudantes de graduação das instituições federais de ensino superior brasileiras. Brasília: Fonaprace. Recuperado de: http://www.andifes.org.br/wp-content/ uploads/2017/11/Pesquisa-de-Perfil-dos-Graduanso-dasIFES_2014.pdf. Acesso em 18 set. 2017.

Gil, A. C. (1999). Métodos e técnicas de pesquisa social. (5a ed.). São Paulo: Atlas.

Kienen, N.; Sahão, F. T.; Rocha, L. B.; Ortolan, M. L.; Soares, N. G.; Yoshiy, S. M.; Prieto, T. (2017). Comportamentos prérequisitos do "Estudar textos em contexto acadêmico". Ces Psicología, 10(2), 28-49. doi: 10.21615/cesp.10.2.3

Kubo, O. M.; Botomé, S. P. (2001). Ensino-aprendizagem: Uma interação entre dois processos comportamentais. Interação, 5, 133-170. doi: http://dx.doi.org/10.5380/psi. v5i1.3321

Lazarus, R. S.; Folkman, S. (1984). Stress, appraisal, and coping. New York: Springer.

Mahmoud, J. S. R.; Staten, R. T.; Hall, L. A.; Lennie, T. A. (2012). The relationship among young adult college students' depression, anxiety, stress, demographics, life satisfaction, and coping styles. Issues in Mental Health Nursing, 33(3), 149-156. doi: 10.3109/01612840.2011.632708.

Moreno, P. F.; Soares, A. B. (2014). O que vai acontecer quando eu estiver na universidade?: Expectativas de jovens estudantes brasileiros. Aletheia, 45, 114-127. Recuperado de http://pepsic.bvsalud.org/pdf/aletheia/n45/n45a09.pdf

Oliveira, C. T.; Carlotto, R. C.; Vasconcelos, S. J. L.; Dias, A. C. G. (2014). Adaptação acadêmica e coping em estudantes universitários brasileiros: Uma revisão de literatura. Revista Brasileira de Orientação Profissional, 15(2), 177186. Recuperado de http://pepsic.bvsalud.org/pdf/rbop/ v15n2/08.pdf

Oliveira, C. T. de; Santos, A. S. dos; Dias, A. C. G. (2016). Expectativas de universitários sobre a universidade: sugestões para facilitar a adaptação acadêmica. Revista Brasileira de Orientação Profissional, 17(1), 43-53. Recuperado de http://pepsic.bvsalud.org/pdf/rbop/ v17n1/06.pdf 
Oliveira, C. T.; Dias, A. C. G. (2014). Dificuldades na trajetória universitária e rede de apoio de calouros e formandos. Psico, 45(2), 187-97. doi https://doi.org/10.15448/19808623.2014.2.13347

Padovani, R. C.; Neufeld, C. B.; Maltoni, J.; Barbosa, L. N. F.; Souza, W. F.; Cavalcanti, H. A. F.; Lameu, J. N. (2014). Vulnerabilidade e bem-estar psicológicos do estudante universitário. Revista Brasileira de Terapias Cognitivas, 10(1), 02-10. doi:10.5935/1808-5687.20140002.

Persaud, N.; Persaud I. (2016). The Relationship between socio-demographics and stress levels, stressors, and coping mechanisms among undergraduate students at a university in Barbados. International Journal of Higher Education, 5(1), 11-27. doi:10.5430/ijhe.v5n1p11.

Santos, G. C. V.; Kienen, N.; Viecili, J.; Botomé, S. P.; Kubo, O. M. (2009). "Habilidades" e "Competências" a desenvolver na capacitação de psicólogos: Uma contribuição da Análise do Comportamento para o exame das diretrizes curriculares. Interação em Psicologia, 13(1), 131-145.

Skinner, B. F. (1974). About behaviorism. New York: Alfred A. Knopf.
Stoliker, B. E.; Lafreniere, K. D. (2015). The influence of perceived stress, loneliness, and learning burnout on university students' educational experience. College Student Journal, 49(1), 146-160. Recuperado de https:// eric.ed.gov/?id=EJ1095547

Teixeira, M. A. P.; Bardagi, M. P.; Hutz, C. S. (2007). Escalas de exploração vocacional (EEV) para universitários. Psicologia em Estudo, 12(1), 195-202. doi:10.1590/S141373722007000100023

Teixeira, M. A. P.; Dias, A. C. G.; Wottrich, S. H.; Oliveira, A. M. (2008). Adaptação à universidade em jovens calouros. Psicologia Escolar e Educacional, 12(1), 185-202. doi:10.1590/S1413-85572008000100013.

Withey, L.; Fox, C. L.; Hartley, J. (2014). 'I cannot mess this up anymore': The experiences of undergraduates who withdraw and start again elsewhere. Psychology Teaching Review, 20(1), 78-89. Recuperado de https://files.eric. ed.gov/fulltext/EJ1149779.pdf

Yoshiy, S. M.; Kienen, N. (2018). Gerenciamento de tempo: Uma interpretação analítico-comportamental. Psicologia da Educação, 47(2), 67-77. doi: 10.5935/2175-3520.2018001

Recebido: 20 de maio de 2019 Aprovado: 10 de outubro de 2020

O presente trabalho foi realizado com apoio da Coordenação de Aperfeiçoamento de Pessoal de Nível Superior Brasil (CAPES) - Código de Financiamento 001.

Este artigo faz parte da dissertação de mestrado da primeira autora, sob orientação da coautora. 